\title{
Automatic tracking of swimming microorganisms in 4D digital in-line holography data $\dagger$
}

\author{
L. Leal Taixé1,*, M. Heydt ${ }^{2}$, A. Rosenhahn ${ }^{2}$, and B. Rosenhahn ${ }^{1}$ \\ ${ }^{1}$ Leibniz Universität Hannover, Appelstr. 9A, Hannover, Germany \\ ${ }^{2}$ Applied Physical Chemistry, University of Heidelberg, INF 253, Heidelberg, Germany
}

\begin{abstract}
Digital in-line holography is a microscopy technique which has gotten an increasing amount of attention over the last few years in the fields of microbiology, medicine and physics, as it provides an efficient way of measuring $3 D$ microscopic data over time. In this paper we approach the challenges of a high throughput analysis of holographic microscopy data and present a system for detecting particles in $3 D$ reconstructed holograms and their $3 D$ trajectory estimation over time. Our main contribution is a robust method which evolves from the Hungarian bipartite weighted graph matching algorithm and allows us to deal with newly entering and leaving particles and compensate for missing data and outliers. In the experiments we compare our fully automatic system with manually labeled ground truth data and we can report an accuracy between $76 \%$ and $91 \%$.
\end{abstract}

\section{Introduction}

Most processes in biology are three dimensional. This holds true for the structure of biological matter and for interaction of even the smallest building blocks of nature. In the case of small swimming objects, such as bacteria, algae or a range of pathogens, motility is of fundamental importance as interactions often are controlled by their motility. Motion and interaction with the environment is the prerequisite for e.g. formation of biofilms [22], biofouling by marine algae $[8,20]$ or the action of pathogens [6]. Understanding the motility and behavioral patterns of microorganisms allows us to understand their interaction with the environment and thus control unwanted consequences such as infections or biofouling. Many microorganisms are some micrometer large and swim in highly complex, 3D motion patterns

\footnotetext{
*Corresponding author: L. Leal Taixé, email: leal@tnt.uni-hannover.de

${ }^{\dagger}$ This work was partially funded by the German Research Foundation, DFG projects RO 2497/7-1 and RO 2524/2-1 and the EU project AMBIO.
}

with velocities of multiple body length per second. Due to their small size they usually swim in a highly laminar, low Reynolds number regime.

Microscopes are necessary to study such small objects at high magnification. Berg built a tracking light microscope, capable of tracking one bacterium at a time in 3D by using translation stages, which has been used to investigate bacteria like Escherichia Coli [2]. Another way of measuring 3D trajectories is stereoscopy, which requires two synchronized cameras [1]. Confocal microscopy has also been used to study the motion of particles in colloidal systems over time, however the nature of this scanning technique limits the achievable frame rate [25].

For any of these techniques, in order to draw statistically relevant conclusions, thousands of images have to be analyzed. Nowadays, this analysis is still heavily dependent on manual intervention. The search for a nearly automatic analysis of biological images has been extensively studied [16] but most of the work focuses on position as well as on the shape of the particle [24]. Statistical methods like Kalman and particle filters are widely used for tracking but they need a dynamical model of the target, a task that can be challenging depending on the microorganism under study, e.g., the recent work [12] presents a complete vision system for $2 \mathrm{D}$ cell tracking. It proves the increasing demand for efficient computer vision approaches in the field of microscopy as an emerging discipline. In contrast to [12], we deal with 3D trajectories of moving particles over time. In this work we further propose a global optimal matching solution and not a local one as suggested in [14].

In this paper, we present a microscopy technique, Digital in-line holography, which provides videos of a 3D volume and is used to study complex movements of microorganisms. The huge amount of information that we can extract from holographic images has driven us to create a new method to analyze this complex 4D data. We propose a geometrically motivated and globally optimal multi-level Hun- 
garian to compensate for leaving and entering particles, recover from missing data and erase the outliers to reconstruct the whole trajectory of the microorganisms.

This paper is organized as follows: Section 2 offers a description of the Digital in-line holography technique. In Section 3 we introduce the Hungarian algorithm, in Section 4 the creation of the cost matrix is explained, along with two modifications. The main contribution of our paper, the multi-level Hungarian, is detailed in Section 5. Section 6 includes relevant experimental results to show the performance of our algorithm.

\section{Digital in-line holography}

Digital in-line holography provides an alternative, lensless microscopy technique which intrinsically contains three dimensional information about the investigated volume. It does not require a feedback control which responds to the motion and it uses only one CCD chip. This makes the method very straightforward and can be implemented with a very simple setup as shown in Figure 1.

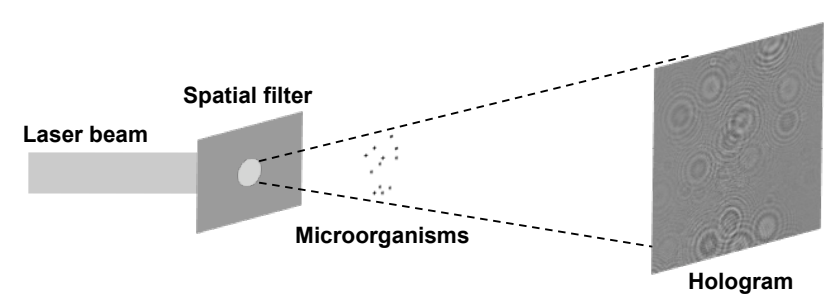

Figure 1: Schematic setup for a digital in-line holographic experiment consisting of the laser, a spatial filter to create the divergent light cone, the objects of interest (e.g. microorganisms) and a detector which records the hologram

The holographic microscope requires only a divergent wavefront which is produced by diffraction of laser light from a pinhole. A CCD chip finally captures the hologram. The holographic microscope setup follows directly Gabors initial idea [4] and has been implemented for laser radiation by $\mathrm{Xu}$ et al. [26]. A hologram recorded without the presence of particles, called the source is subtracted from each hologram. This is used to reduce the constant illumination background and other artifacts; there are filtering methods $[3,19]$ to achieve this in case a source image is not readily available. These resulting holograms can then be reconstructed back into real space by a Kirchhoff-Helmholtz transformation [26] shown in Equation 1.

$$
K(\mathbf{r})=\int_{S} d^{2} \xi I(\xi) e^{\frac{2 \pi i \xi \cdot \mathbf{r}}{\lambda \xi}}
$$

in which the integration extends over the 2-D surface of the screen with coordinates $\xi=(X, Y, L)$, where $L$ is the distance from the source (pinhole) to the center of the screen (CCD chip), and $I(\xi)$ is the contrast image (hologram) on the screen obtained by subtracting the images with and without the object present (so called source image). Several reconstructing methods are evaluated in [9].

From the reconstruction we obtain 3 projections $X Y$, $X Z$ and $Y Z$, shown in Figure 2, as described in [7].

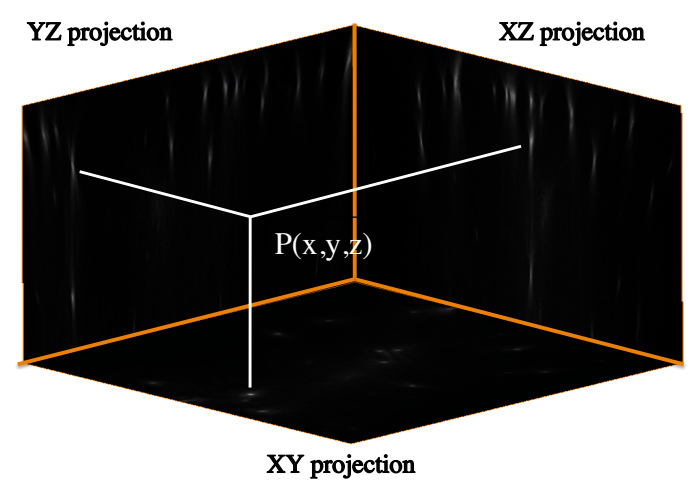

Figure 2: Projections XY, XZ and YZ obtained after reconstructing the holograms. $\mathrm{P}(\mathrm{x}, \mathrm{y}, \mathrm{z})$ represents the $3 \mathrm{D}$ position of the microorganisms [7]

The great advantage is that every object located in the light cone between the pinhole and the CCD detector can be reconstructed and thus located in three dimensions. Using video sequences of holograms, it is possible to track objects in 3D over time and multiple spores present in a single frame can be tracked simultaneously $[5,8,14]$. Using this advantage of digital in-line holography a number of 3D phenomena in microbiology have been investigated: Lewis et al. [11] examined the swimming speed of Alexandrium (Dinophyceae), Sheng et al. [21] studied the swimming behavior of predatory dinoflagellates in the presence of prey, and Sun et al. [23] used a submersible device to investigate in situ plankton in the ocean.

\section{Hungarian: the matching algorithm}

Graph Matching is one of the fundamental problems in Graph Theory and it can be defined as: given a graph $G=$ $(V, E)$, where $E$ represents its set of edges and $V$ its set of nodes, a matching $M$ in $G$ is a set of pairwise non-adjacent edges, which means that no edges share a common vertex.

A graph $G=(V, E)$ is bipartite when its vertices $V$ can be divided into two sets $X$ and $Y$ such that: the original set of vertices is formed by the union of the two subsets ( $V=X \cup Y$ ); each vertex belongs to just one of the two subsets $(X \cap Y=\emptyset)$; every edge in $X$ connects to one in $Y(E \subseteq X \times Y)$. A matching in a bipartite graph is always perfect, that is, all the vertices are matched. It is easier to compute than in general graphs, as the number of possible combinations decreases considerably with the bipartite re- 
striction and the result is found without approximations.

For our application, we are specially interested in the Assignment Problem, which consists in finding a maximum weight matching in a weighted bipartite graph. In a general form, the problem can be expressed as: "There are $\mathrm{N}$ jobs and $\mathrm{N}$ workers. Any worker can be assigned to any job, incurring some cost that varies depending on the job-worker assignment. All jobs must be performed by assigning exactly one worker to each job in such a way that the total cost is minimized (or maximized)". For the subsets of vertices $X$ and $Y$, we build a cost matrix in which the element $C(i, j)$ will represent the weight or cost related to vertex $i$ in $X$ and vertex $j$ in $Y$.

For numerical optimization we use the Hungarian or Munkres' assignment algorithm, a combinatorial optimization algorithm $[10,17]$ that solves the bipartite graph matching problem in polynomial time. For implementation details on the Hungarian we recommend [18]. Our initial problem configuration is: there are $M$ particles in frame $t_{1}$ and $N$ particles in frame $t_{2}$. The Hungarian will help us to find which particle in $t_{1}$ corresponds to which particle in $t_{2}$, allowing us to reconstruct their full trajectories in 3D space. Nonetheless, the Hungarian algorithm has some disadvantages which we should know and correct to make the algorithm more robust. In the context of our project, we summarize in Table 1 some of the advantages and disadvantages of the Hungarian algorithm.

\begin{tabular}{l|l}
\hline ADVANTAGES & DISADVANTAGES \\
\hline $\begin{array}{l}\text { Finds a global solution for } \\
\text { all vertices }\end{array}$ & $\begin{array}{l}\text { Cannot handle missing } \\
\text { vertices (a) }\end{array}$ \\
\hline Cost matrix is versatile & $\begin{array}{l}\text { Cannot handle entering or } \\
\text { leaving particles (b) }\end{array}$ \\
\hline $\begin{array}{l}\text { Easy to solve, bipartite } \\
\text { matching is the simplest } \\
\text { of all graph problems }\end{array}$ & $\begin{array}{l}\text { No discrimination of } \\
\text { matches even if the cost is } \\
\text { very high (c) }\end{array}$ \\
\hline
\end{tabular}

Table 1: Summary of the advantages and disadvantages of the Hungarian algorithm

In the following sections, we present how to solve the three disadvantages: (a) is solved with the multi-level Hungarian method explained in Section 5, (b) is solved with the IN/OUT states of Section 4.1 and finally a solution for (c) is presented in Section 4.2 as a maximum cost restriction.

\section{Cost function}

The cost function $C$ as key input for the Hungarian algorithm is created using the Euclidean distances between particles, that is, element $C(i, j)$ of the matrix represents the distance between particle $i$ of frame $t_{1}$ and particle $j$ of frame $t_{2}$. With this matrix, we need to solve a minimum as- signment problem since we are interested in matching those particles which are close to each other.

Note that it is also possible to include in the cost function other characteristics of the particle like speed, size or gray level distribution. Such parameters can act as additional regularizers during trajectory estimation.

\subsection{IN and OUT states}

In order to include more knowledge about the environment to the Hungarian algorithm and avoid matches with very high costs, we have created a variation for the cost matrix. The idea is to model the possible entrances and exits of the particles from the Field Of View (FOV) of the holographic microscope. We include the information of the environment by calculating the distance of each particle to the nearest edge of the FOV as in Equation 2, where $m_{x}, m_{y}, m_{z}$ are the low borders and $M_{x}, M_{y}, M_{z}$ are the high borders for each of the axis. Note that the low border in the $z$ axis is not included as it represents the surface where the microorganisms might settle and, therefore, no particles can enter or leave from there. If $N$ is the number of particles in frame $t_{2}$, then $k>N$.

$$
\begin{aligned}
& C(i, k)=\min \left(\left|P_{i}-M_{x}\right|,\left|P_{i}-m_{x}\right|,\right. \\
& \left.\quad\left|P_{i}-M_{y}\right|,\left|P_{i}-m_{y}\right|,\left|P_{i}-M_{z}\right|\right)
\end{aligned}
$$

If the distance is small enough, the Hungarian algorithm matches the particle with an IN/OUT state.

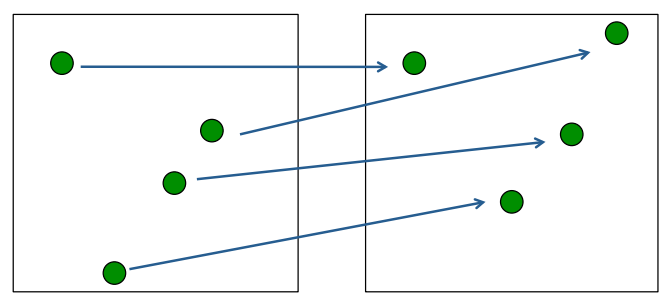

(a) Wrongly matched

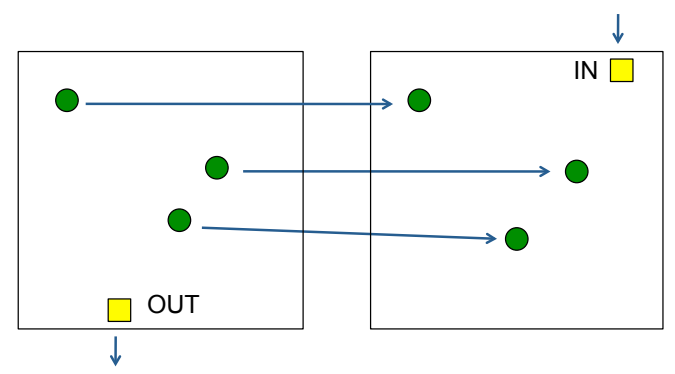

(b) Correctly matched as a result of the IN/OUT state feature

Figure 3: Representation of the particles in frame $t_{1}$ (left) and $t_{2}$ (right). The lines represent the matchings.

In Figure 3 we consider the simple scenario in which we have 4 particles in one frame and 4 in the next frame. As 
we can see, there is a particle which leaves the scene from the edge below and a particle which enters the scene in the next frame from the edge above. As shown in Figure 3a, the Hungarian algorithm finds a wrong matching since the result is completely altered by the entering/leaving particles. With the introduction of the IN/OUT state feature, the particles are now correctly matched (see Figure 3b) and the ones which enter/leave the scene are identified as independent particles.

\subsection{Maximum cost restriction}

Theoretically, particles can only enter/leave the scene by crossing the borders of the FOV, but due to noise and illumination irregularities of the holograms, it is possible for particles to disappear in the middle of the scene. If a particle is no longer detected, all the matches can be greatly affected in a similar way as shown in Figure 3a. That is why we introduce a maximum cost restriction for the cost matrix, which does not allow matches which have costs higher than a given threshold. This threshold is based on the observed maximum speed of the algae spores under study [7].

\section{Multi-level Hungarian for missing data}

In this section we present our main contribution, the multi-level Hungarian. The whole method described in Section 5 is summarized in a diagram in Figure 4.

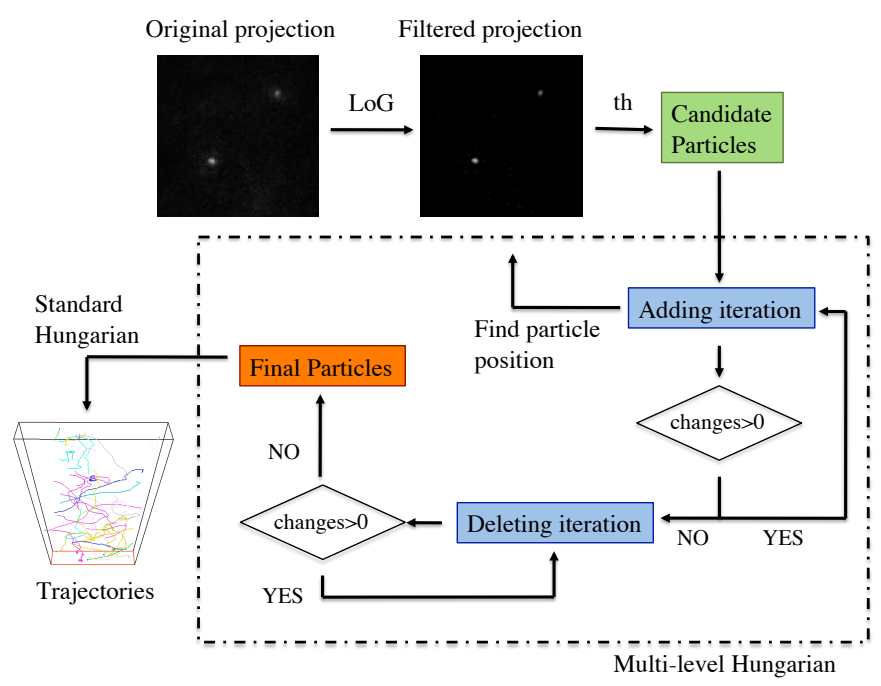

Figure 4: Diagram of the algorithm described in Section 5

\subsection{Initial particle detection}

In our sequences we are observing the algae Ulva linza, which has a spheric head and four thin flagella. Since the head scatters most of the light, in the projected images the particles have a circular shape. In order to preserve and enhance particles but reduce noise and illumination irregularities of the image, we apply the Laplacian of Gaussian filter (LoG) which, for its shape, is a blob detector [13].

Due to the divergent nature of the light cone, the particles can appear smaller or larger in the projections. Therefore, the LoG filter is applied in several scales [13]. Note that the whole algorithm is extremely adaptable, since we can detect particles with any shape by just changing the filter. The final 3D position (Figure 4, green box labeled "Candidate particles") is determined by thresholding each projection $X Y$, $X Z$ and $Y Z$ to find the particles in each image and crossing the information of the three projections.

\subsection{Compensating for missing data and outliers}

If we consider just the particles detected using the thresholding, we see that there are many gaps within a trajectory (see Figure 7a). These gaps can be a result of morphing (different object orientations yield to different contrast), changes in the illumination, etc. The standard Hungarian is not capable of filling in the missing data and creating full trajectories, therefore, we now introduce a method based on the standard Hungarian that allows us to treat missing data, outliers and create complete trajectories. The general routine of the algorithm, the multi-level Hungarian, is:

- Find the matchings between particles in frames $[i-$ $2 \ldots i+2$ ], so we know the position of each particle in each of these frames (if present). (Section 5.2.1).

- Build a table with all these positions and fill the gaps given some strict conditions. Let the algorithm converge until no particles are added. (Section 5.2.2).

- On the same table and given some conditions, erase the outliers. Let the algorithm converge until no particles are deleted. (Section 5.2.2).

\subsubsection{The levels of the multi-level Hungarian}

The multi-level Hungarian takes advantage of the temporal information in 5 consecutive frames and is able to recover from occlusions and gaps in up to two consecutive frames. The standard Hungarian gives us the matching between the particles in frame $t_{1}$ and frame $t_{2}$ and we use this to find matchings of the same particle in 5 consecutive frames, $[i-2, \ldots, i+2]$. In order to find these matchings, the Hungarian is applied on different levels. The first two levels, represented in Figure 5 by red arrows, are created to find the matching of the particles in the frame of study, frame $i$. But it can also be the case that a particle is not present in frame $i$ but is present in the other frames. To solve all the possible combinations given this fact, we use Levels 3, 4 and 5, represented in Figure 5 by green arrows. 


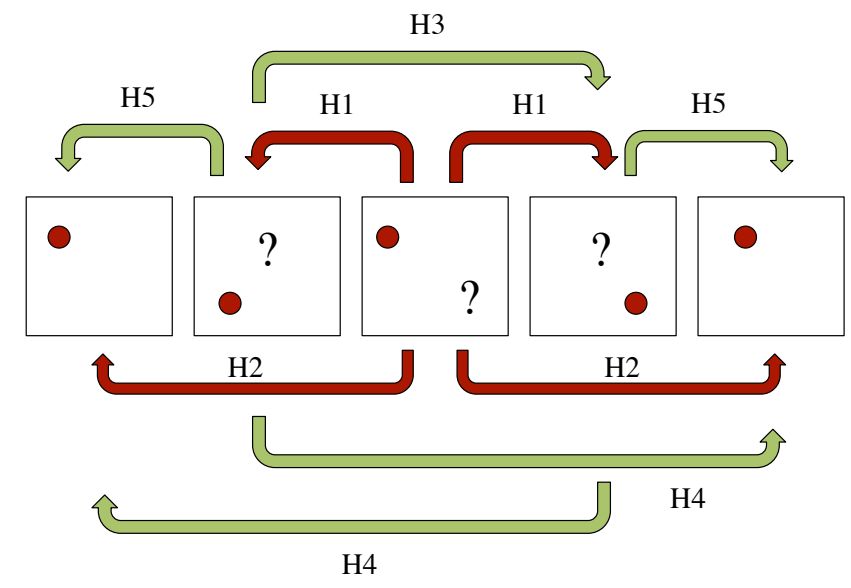

Figure 5: Represented frames: [i-2,i-1,i,i+1,i+2]. Levels of the multi-level Hungarian.

Below we show a detailed description and purpose of each level of the multi-level Hungarian:

- Level 1: Matches particles in frame $i$ with frames $i \pm 1$.

- Level 2: Matches particles in frame $i$ with frames $i \pm 2$. With the first two levels, we know, for all the particles in frame $i$, their position in the neighboring frames (if they appear).

- Level 3: Matches particles in frame $i-1$ with frame $i+1$.

- Level 4: Matches particles in frame $i \pm 1$ with frame $i \mp$ 2. Level 3 and 4 solve the detection of matchings when a particle appears in frames $i \pm 1$ and might appear in $i \pm 2$, but is not present in frame $i$.

- Level 5: Matches particles in frame $i \pm 1$ with frame $i \pm 2$.

Of course, the maximum cost is proportional to the difference of frames that the Hungarian matches, e.g. if Level 1 has cost $C_{1}$, Level 2 has cost $2 C_{1}$ and Level 4 cost $3 C_{1}$.

\subsubsection{Conditions to add/delete particles}

Once all the levels are applied hierarchically, a table with the matching information is created. On one axis we have the number of particles and on the other the 5 frames from $[i-2 \ldots i+2]$, as shown in Figure 6.

To change the table information, we use two iterations: the adding iteration and the deleting iteration which appear in Figure 4 as blue boxes. During the adding iteration, we look for empty cells in the table where there is likely to be a particle. A new particle position is added if, and only if, two conditions are met:
1. There are at least 3 particles present in the row. Particles have continuity while noise points do not.

2. It is not the first or last particle of the row. We use this strict condition to avoid the creation of false particle positions or the incorrect elongation of trajectories.

Let's look at particle 6 of the table in Figure 6. In this case, we do not want to add any particle in frames $i-2$ and $i-1$, since the trajectory could be starting at frame $i$. In the case of particle 4 , we do not want to add a particle in frame $i+2$ because the trajectory could be ending at $i+1$. Each iteration repeats this process for all frames, and we iterate until the number of particles added converges.

After convergence, the deleting iteration starts and we erase the outliers considered as noise. A new particle position is deleted if, and only if, two conditions are met:

1. The particle is present in the frame of study $i$.

2. There are less than 3 particles in the same row.

We only erase particles from the frame of study $i$ because it can be the case that a particle appears blurry in the first frames but is later correctly detected and has more continuity. Therefore, we only delete particles from which we know the complete neighborhood. Each iteration repeats this process for all frames, and we iterate until the number of particles deleted converges. The resulting particles are shown in Figure 6.

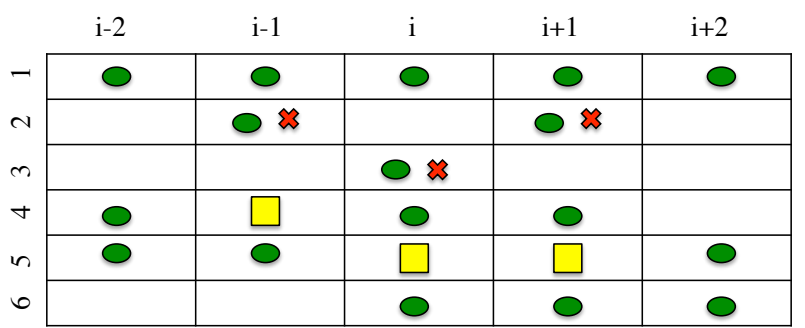

Figure 6: Table with: the initial particles detected by the multi-level Hungarian (green ellipses), the ones added in the adding iteration (yellow squares) and the ones deleted in the deleting iteration (red crosses). In the blank spaces no position has been added or deleted.

\subsubsection{Missing data interpolation}

During the adding iteration, in order to find the correct position of the new particle, we use the information of the filtered projection (Figure 4). For example, if we are looking to add a particle in frame $i-1$, we go to the filtered projections $\mathrm{XY}, \mathrm{XZ}, \mathrm{YZ}$ in $t=i-1$, take the position of the corresponding particle in $t=i$ or $t=i-2$ and search 
for the maximum value within a window $w$. If the position found is already present in the candidate particles' list of that frame, we go back to the projection, erase that particle, and find the position of the second maximum value. This allows us to distinguish two close particles.

There are many studies on how to improve the particle depth-position resolution ( $z$-position). As in [15] we use the traditional method of considering the maximum value of the particle as its center. Other more complex methods [3] have been developed which also deal with different particle sizes, but the flexibility of using morphological filtering already allows us to easily adapt our algorithm.

\subsection{The final Hungarian}

Once the final particle positions are obtained (in Figure 4, orange box labeled "Final particles"), we perform one last step to determine the trajectories. We use the standard Hungarian to match particles from frame $i$ to frame $i+1$.

\section{Experimental results}

In order to test our algorithm we use 6 sequences (labeled S1 to S6) in which the swimming motion of Ulva linza spores is observed [8]. All the sequences have some particle positions which have been semi-automatically reconstructed and manually labeled and inspected (our ground truth) for later comparison with our fully-automatic results.

\subsection{Performance of the standard Hungarian}

First of all, we want to show the performance of the final standard Hungarian appearing in Section 5.3. For this, we use the ground truth particle positions and apply the Hungarian algorithm to find the matchings and therefore the complete trajectories. If we compare the automatic matches to the ground truth, we can see that in $67 \%$ of all the sequences, the number of particles is correctly detected, while in the remaining $33 \%$, there is just a $5 \%$ difference in the number of particles. The average accuracy of the matchings reaches $96.61 \%$.

To further test the robustness of the Hungarian algorithm, we add random noise to each position of our particles. The added noise is the one intrinsically present in this imaging technique, determined experimentally in [7]. $N=100$ experiments are performed on each of the surfaces and the accuracy is recorded. Results show that the average accuracy of the matching is just reduced from $96.61 \%$ to $93.51 \%$, making the Hungarian algorithm very robust to the noise present in the holographic images and therefore perfectly suited to find the trajectories of the particles.

\subsection{Performance of the multi-level Hungarian}

To test the performance of the multi-level Hungarian we apply the Final Hungarian on three sets of particles:
- Set A: particles determined by the threshold (pre multilevel Hungarian)

- Set B: particles corrected after multi-level Hungarian

- Set C: ground truth particles

We then start by comparing the number of particles detected, as shown in Table 2.

\begin{tabular}{lllllll}
\hline & S1 & S2 & S3 & S4 & S5 & S6 \\
\hline Set A & 1599 & 1110 & 579 & 668 & 1148 & 2336 \\
\hline Set B & 236 & 163 & 130 & 142 & 189 & 830 \\
\hline Set C & 40 & 143 & 44 & 54 & 49 & 48 \\
\hline
\end{tabular}

Table 2: Comparison of the number of particles detected

The number of particles detected in Set A is drastically reduced in Set B, after applying the multi-level Hungarian, demonstrating its abilities to compensate for missing data and merging trajectories. If we compare it to Set $\mathrm{C}$, we see that the number is still too high, indicating possible tracks which were not merged and so detected as independent.

Nonetheless, as we do not know exactly the amount of particles present in a volume (not all particle positions have been labeled), it is of great value for us to compare the average length of the trajectories, defined as the number of frames in which the same particle is present. The results are shown in Table 3 where we can clearly see that the average length of a trajectory is greatly improved with the multilevel Hungarian, which is crucial since long trajectories give us more information on the behavior of the particles.

\begin{tabular}{lllllll}
\hline & S1 & S2 & S3 & S4 & S5 & S6 \\
\hline Set A & 3 & 5 & 5 & 4 & 6 & 7 \\
\hline Set B & 19 & 31 & 27 & 23 & 38 & 23 \\
\hline Set C & 58 & 54 & 54 & 70 & 126 & 105 \\
\hline
\end{tabular}

Table 3: Comparison of the trajectories' average length

If we consider just useful trajectories for particle analysis, that is, trajectories with a length of more than 25 frames, the multi-level Hungarian allows us to extract $10 \%$ more of useful trajectories out of each analyzed volume, as shown in Table 4.

\begin{tabular}{lll}
\hline Set A & Set B & Set C \\
\hline $20.7 \%$ & $30.1 \%$ & $69 \%$ \\
\hline
\end{tabular}

Table 4: Average percentage of useful trajectories 


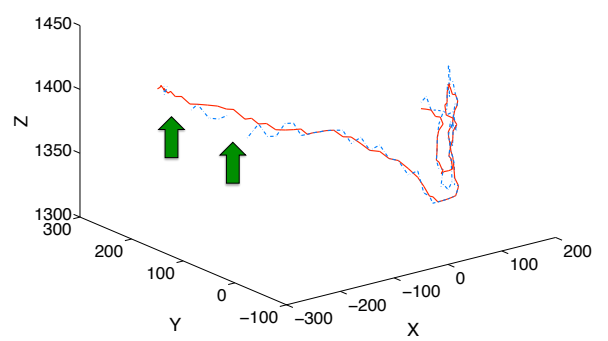

(a)

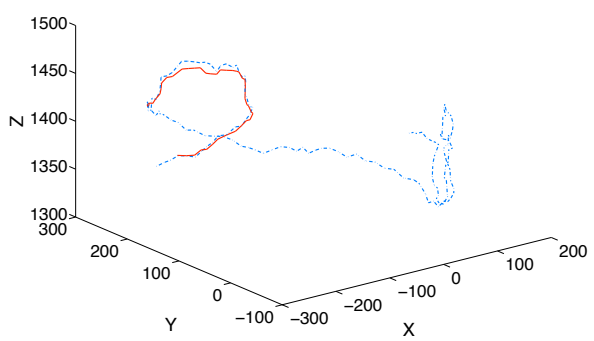

(b)

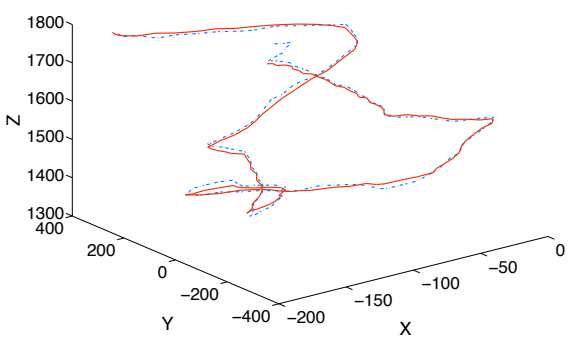

(c)

Figure 7: (a) 3 separate trajectories are detected with the standard Hungarian (blue dashed line). Merged trajectory detected with our method (with a smoothing term, red line). Missing data spots marked by arrows. (b),(c) Ground truth trajectories (blue dashed line). Trajectories automatically detected with our method (red line).

Ultimately, this means that fewer volumes have to be analyzed in order to have enough information to draw conclusions of the behavior of a particle.

\subsection{Performance of the global method}

Finally, we are interested in implementing the global method on the original projections and compare the overall results with the ground truth data. For this comparison, we are going to present two values:

- Missing: percentage of ground truth particles which are not present in the automatic determination

- Extra: percentage of automatic particles that do not appear in the ground truth data

In Table 5 we show the detailed results for each surface.

\begin{tabular}{lllllll}
\hline & S1 & S2 & S3 & S4 & S5 & S6 \\
\hline Missing (\%) & 8.9 & 20.7 & 19.1 & 23.6 & 11.5 & 12.9 \\
& & & & & & \\
\hline Extra (\%) & 54.9 & 34.1 & 46.5 & 13.3 & 25.8 & 74.6
\end{tabular}

Table 5: Missing labeled and extra automatic particles

Our automatic algorithm detects between $76 \%$ and $91 \%$ of the particles present in the volume. This gives us a measure of how reliable our method is, since it is able to detect most of our verified particle positions. Putting this information together with the percentage of particles detected by our algorithm but not labeled, we can see that our method extracts much more information from the volume of study. This is clear in the case of S6, where we have a volume with many crossing particles difficult to manually label and where our algorithm gives us almost $75 \%$ more information.

We now consider the actual trajectories and particle position and measure the position error of our method. The error is measured as the Euclidean distance between each point of the ground truth and the automatic trajectories, both at time $t$. In Figure $7 \mathrm{a}$ we can see the 3 independent trajectories found with the standard Hungarian and the final merged trajectory which proves the power of our algorithm to fill in the gaps (pointed by arrows). In Figure $7 \mathrm{~b}$ we can see that the automatic trajectory is much shorter (there is a length difference of 105 frames), although the common part is very similar with an error of just 4.2. Figure $7 \mathrm{c}$ on the other hand, shows a perfectly matched trajectory with a length difference of 8 frames and error of 6.4 for the whole trajectory. This proves the determination of the particle position is accurate but the merging of trajectories can be improved.

Finally, we present in Figure 8 a view of the complete volume with the trajectories of the particles found in a fully automatic way. We just represent particles with length over 40 frames to visualize the independent tracks easily.

\section{Conclusions}

We have presented an automatic method to track multiple swimming microorganisms in 4D digital in-line holography data. This imaging technique provides images of a 3D volume in time, which allows us to recover full trajectories of microorganisms. We have focused our study on a powerful matching tool, the Hungarian-Munkre's matching algorithm, to create a robust method that is able to overcome the few disadvantages of the standard Hungarian. The new multi-level Hungarian recovers from missing data, discards outliers and is able to incorporate geometrical information in order to account for entering and leaving particles. The performance of each step of the method is carefully tested. The automatically determined trajectories are compared with ground truth data, proving the method detects between $75 \%$ and $90 \%$ of the labeled particles. Our system is proved to be a helpful tool for biologists and physicists to study microorganisms in an easy and fast way, providing a vast amount of analyzed data. 


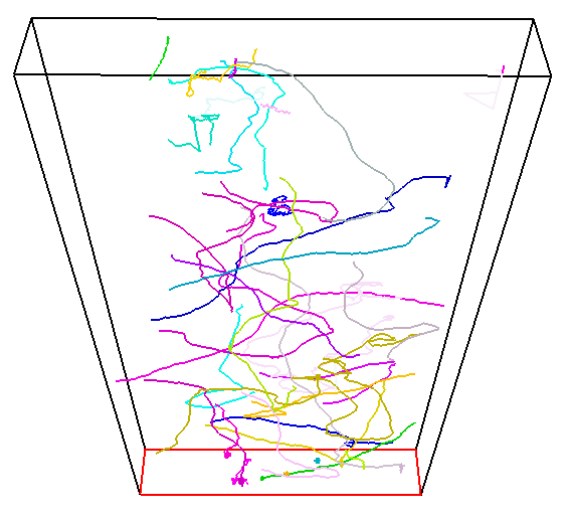

Figure 8: Visualization tool for the volume under study. The surface where spores can settle is marked in red.

\section{References}

[1] S. Baba, S. Inomata, M. Ooya, Y. Mogami, and A. Izumikurotani. 3-dimensional recording and measurement of swimming paths of microorganisms with 2 synchronized monochrome cameras. Review of Scientific Instruments, 62(2):540-541, 1991.

[2] P. Frymier, R. Ford, H. Berg, and P. Cummings. 3dimensional tracking of motile bacteria near a solid planar surface. Proceedings of the National Academy of Sciences of the United States of America, 92(13):6195-6199, 1995.

[3] J. Fugal, T. Schulz, and R. Shaw. Practical methods for automated reconstruction and characterization of particles in digital in-line holograms. Measurement Science and Technology, 20(7):075501, 2009.

[4] D. Gabor. A new microscopic principle. Nature, 161(8):777, 1948.

[5] J. Garcia-Sucerquia, W. Xu, S. Jericho, M. H. Jericho, I. Tamblyn, and H. Kreuzer. Digital in-line holography: 4$\mathrm{d}$ imaging and tracking of microstructures and organisms in microfluidics and biology. Proceedings of the SPIE, 6026:267-275, 2006.

[6] M. Ginger, N. Portman, and P. McKean. Swimming with protists: perception, motility and flagellum assembly. Nature Reviews Microbiology, 6(11):838-850, 2008.

[7] M. Heydt, P. Divós, M. Grunze, and A. Rosenhahn. Analysis of holographic microscopy data to quantitatively investigate three dimensional settlement dynamics of algal zoospores in the vicinity of surfaces. The European Physical Journal E: Soft Matter and Biological Physics, 2009.

[8] M. Heydt, A. Rosenhahn, M. Grunze, M. Pettitt, M. E. Callow, and J. A. Callow. Digital in-line holography as a three-dimensional tool to study motile marine organisms during their exploration of surfaces. The Journal of Adhesion, 83(5):417-430, 2007.

[9] T. Kreis, M. Adams, and W. Juptner. Methods of digital holography: a comparison. Proceedings of the SPIE, 3098:224-33, 1997.

[10] H. Kuhn. The hungarian method for the assignment problem. Naval Research Logistics, 2:83-87, 1955.
[11] N. I. Lewis, W. Xu, S. Jericho, H. Kreuzer, M. Jericho, and A. Cembella. Swimming speed of three species of alexandrium (dinophyceae) as determined by digital in-line holography. Phycologia, 45(1):61-70, 2006.

[12] K. Li, E. Miller, M. Chen, T. Kanade, L. Weiss, and P. Campbell. Cell population tracking and lineage construction with spatiotemporal context. Medical Image Analysis, 12(5):546566, October 2008.

[13] T. Lindeberg. Scale-space theory in computer vision. Springer, 1994.

[14] J. Lu, J. Fugal, H. Nordsiek, E. Saw, R. Shaw, and W. Yang. Lagrangian particle tracking in three dimensions via singlecamera in-line digital holography. New Journal of Physics, 10, 2008.

[15] N. Masuda, T. Ito, K. Kayama, H. Kono, S. Satake, T. Kunugi, and K. Sato. Special purpose computer for digital holographic particle tracking velocimetry. Optics Express, 14:587-92, 2006.

[16] K. Miura. Tracking movement in cell biology. Microscopy Techniques, pages 267-295, 2005.

[17] J. Munkres. Algorithms for the assignment and transportation problems. Journal of the Society of Industrial and Applied Mathematics, 5(1):32-38, 1957.

[18] R. Pilgrim. Munkres' assignment algorithm; modified for rectangular matrices. Course Notes, Murray State University. http://csclab.murraystate.edu/bob.pilgrim/445/munkres.html.

[19] S. Raupach, H. Vossing, J. Curtius, and S. Borrman. Digital crossed-beam holography for in situ imaging of athmospheric particles. Journal of Optics A: Pure and Applied Optics, 8:796-806, 2006.

[20] A. Rosenhahn, T. Ederth, and M. Pettitt. Advanced nanostructures for the control of biofouling: The fp6 eu integrated project ambio. Biointerphases, 3(1):IR1-IR5, 2008.

[21] J. Sheng, E. Malkiel, J. Katz, J. Adolf, R. Belas, and A. Place. Digital holographic microscopy reveals preyinduced changes in swimming behavior of predatory dinoflagellates. Proceedings of the National Academy of Sciences of the United States of America, 104(44):1751217517, 2007.

[22] P. Stoodley, K. Sauer, D. Davies, and J. Costerton. Biofilms as complex differentiated communities. Annual Review of Microbiology, 56:187-209, 2002.

[23] H. Sun, D. Hendry, M. Player, and J. Watson. In situ underwater electronic holographic camera for studies of plankton. IEE Journal of Oceanic Engineering, 32(2):373-382, 2007.

[24] G. Tsechpenakis, L. Bianchi, D. Metaxas, and M. Driscoll. A novel computation approach for simultaneous tracking and feature extraction of c. elegans populations in fluid environments. IEEE Transactions on Biomedical Engineering, 55(5):1539-49, May 2008.

[25] E. Weeks, J. Crocker, A. Levitt, A. Schofield, and D. Weitz. Three-dimensional direct imaging of structural relaxation near the colloidal glass transition. Science, 287(5452):627631, 2000.

[26] W. Xu, M. Jericho, I. Meinertzhagen, and H. Kreuzer. Digital in-line holography for biological applications. Proceedings of the National Academy of Sciences of the United States of America, 98(20):11301-11305, 2001. 